EPJ Web of Conferences 47, 15005 (2013)

DOI: $10.1051 /$ epjconf/20134715005

(C) Owned by the authors, published by EDP Sciences, 2013

\title{
On the Gaia exoplanet discovery potential
}

\author{
A. Sozzettia \\ INAF - Osservatorio Astrofisico di Torino, via Osservatorio 20, 10025 Pino Torinese, Italy
}

\begin{abstract}
In its all-sky astrometric survey, Gaia will be sensitive to giant planets orbiting within a few AUs of hundreds of thousands of bright main-sequence stars within $\approx 200 \mathrm{pc}$. The impact of Gaia observations in the astrophysics of planetary systems cannot be underestimated (e.g., Casertano et al. 2008), particularly if seen in synergy with other techniques for planet detection and characterization (e.g., Sozzetti 2011). I will briefly review the mission status and discuss relevant technical issues associated with the precise and accurate determination of Gaia astrometric orbits of planetary systems. I will then highlight some of the important synergies between Gaia high-precision astrometry and other ongoing and planned, indirect and direct planet-finding and characterization programs, both from the ground and in space, and over a broad range of wavelengths, and provide quantitative examples of such synergies using the sample of nearest stars to the Sun as a proxy.
\end{abstract}

\section{INTRODUCTION}

The state-of-the-art of astrometric techniques is currently set by the milli-arcsecond (mas) precision achieved by the Hipparcos mission. For the purpose of astrometrically detecting planetary-mass companions in orbit around stars in the neighborhood of our Sun, this performance levels are not sufficient, and the literature is populated by several famous "blunders", with unconfirmed and refuted planet discovery announcements by astrometry spanning over seventy years (for a review, see Sozzetti 2010, and references therein).

For astrometry to begin contributing significantly to the fast-developing field of exoplanet astrophysics, a quantum leap of at least two orders of magnitude in positional measurements precision must be obtained. This is indeed no trivial task (e.g., Sozzetti 2005). The first experiment set to demonstrate single-epoch measurement accuracies in the neighborhood of 10 micro-arcseconds ( $\mu$ as) will be the upcoming observatory for global astrometry in space, ESA's Cornerstone Mission Gaia.

\section{GAIA}

In its unbiased all-sky survey, Gaia will monitor astrometrically, during its 5-yr nominal mission lifetime, all point sources (stars, asteroids, quasars, extragalactic supernovae, etc.) in the visual magnitude range $6-20 \mathrm{mag}$, a huge database encompassing $\sim 10^{9}$ objects. Using the continuous scanning principle first adopted for Hipparcos, Gaia will determine the five basic astrometric parameters (two positional coordinates $\alpha$ and $\delta$, two proper motion components $\mu_{\alpha}$ and $\mu_{\delta}$, and the parallax $\pi$ ) for all objects, with end-of-mission precision in the rage 5-15 $\mu$ as, depending on spectral type, at the bright end $(V=6 \mathrm{mag})$ and 200-300 $\mu$ as, depending on spectral type, at the faint end $(V=20 \mathrm{mag})$. Gaia astrometry, complemented by on-board spectrophotometry and (partial) radial velocity information, will have the precision necessary to quantify the early formation, and subsequent dynamical, chemical and

\footnotetext{
ae-mail: sozzetti@oato.inaf .it
}

This is an Open Access article distributed under the terms of the Creative Commons Attribution License 2.0, which permits unrestricted use, distribution, and reproduction in any medium, provided the original work is properly cited. 
star formation evolution of the Milky Way Galaxy. The broad range of crucial issues in astrophysics that can be addressed by the wealth of the Gaia data is summarized by e.g., Perryman et al. (2001).

The Gaia satellite, including the payload, is built by industry (EADS-Astrium, Toulouse, France). ESA is in charge of the mission's operations, while the overall data processing is the mandate of the scientific community, embodied by the Gaia Data Processing and Analysis Consortium (DPAC). The launch schedule is now stabilizing, with the present launch date set in October 2013.

The nominal Intermediate Data Release Scenario has been agreed with inputs from the Data Release Policy and DPAC Operations Plan. It is expected that Science Alerts will be released as soon as possible, while presently a total of 4 intermediate data releases is envisioned, the first occurring at 22 months after launch, and with increasing degree of completeness in the amount of released information. This Scenario has been officially endorsed by the Astronomy Working Group of ESA and used as input to the Announcement of Opportunity for the Archive Access Coordination Unit (CU9) to DPAC, closed in January 2013.

\section{ASTROMETRIC ORBITS OF EXOPLANETS: THE CHALLENGE}

Gaia is ushering us intpo the age of $\mu$ as astrometry, finally enabling secure astrometric detections of planetary-mass companions around nearby stars. However, the improvement of over two orders of magnitude in single-measurement precision with respect to the state-of-the-art mas-precision set by Hipparcos astrometry is not in itself sufficient. There is in fact a variety of technical problems associated with the modeling of the astrometric signatures of planetary systems that must be carefully dealt with.

\subsection{Orbital fits of planetary systems}

The problem of the correct determination of the astrometric orbits of planetary systems using Gaia data (highly non-linear orbital fitting procedures, with a large number of model parameters) will present many difficulties. For example, it will be necessary to assess the relative robustness and reliability of different procedures for orbital fits. Consistency checks between different solution algorithms will be mandatory as a way of learning the lessons of radial-velocity surveys, that are showing us, particularly in the case of multiple-planet systems, how disagreement on orbital solution details, and sometime number of planets!, based on the same datasets is not that uncommon (e.g., Forveille et al. 2011, and references therein; Hatzes et al. 2011, and references therein). A detailed understanding of the statistical properties of the uncertainties associated with the model parameters will have to be developed, based on the relative merit of different metrics tailored to this task, such as covariance matrices, $\chi^{2}$ surface mapping, and bootstrapping procedures. For multiple systems, a trade-off will have to be found between accuracy in the determination of the mutual inclination angles between pairs of planetary orbits, single-measurement precision and redundancy in the number of observations with respect to the number of estimated model parameters. It will constitute a challenge to correctly identify signals (and the associated uncertainties) with amplitude close to the measurement errors, particularly in the presence of larger signals induced by other companions and/or sources of astrophysical noise of comparable magnitude. Finally, in cases of multiple-component systems where dynamical interactions are important (a situation experienced already by radial-velocity surveys), fully dynamical (Newtonian) fits involving an n-body code might have to be used to properly model the Gaia astrometric data and to ensure the short- and long-term stability of the solution (see Sozzetti 2005).

\subsection{Planetary systems in the Gaia data processing pipeline}

All the above issues could have a significant impact on Gaia's capability to detect and characterize planetary systems. For these reasons, within the pipeline of Coordination Unit 4 (object processing) of the Gaia Data Processing and Analysis Consortium (DPAC), in charge of the scientific processing of the 


\section{Hot Planets and Cool Stars}

Gaia data and production of the final Gaia catalogue to be released sometime in 2021, a Development Unit (DU437) has been specifically devoted to the modelling of the astrometric signals produced by planetary systems. The DU is composed of several tasks, which implement multiple robust procedures for (single and multiple) astrometric orbit fitting (such as Markov Chain Monte Carlo and genetic algorithms) and the determination of the degree of dynamical stability of multiple-component systems. I provide here a quick-look view of the software and its status.

\subsection{Orbit fitting algorithms}

Figure 1 shows the activity diagram of DU437. The main feature of this software module consists of the use of two different solution algorithms for fitting astrometric orbits of planetary systems to Gaia data.

The first algorithm is based on a hybrid Markov Chain Monte Carlo (MCMC) approach. The global non-linear least-square problem is partially linearized in then paramters $a_{j}, \omega_{j}, \Omega_{j}$, and $i_{j}$ (for $j=1, \ldots, n$, where $n$ is the number of planets) using the Thiele-Innes elements representation (Green 1985). An iterative period search provides seeds for initializing the MCMC procedure (a paralleltempering algorithm upgrade is in the works). An MCMC chain is then run on the non linear parameters $\left(e_{j}, P_{j}, \tau_{j}\right)$, drawing from Gaussian independent distributions. Each step of the chain drives a linear LSQ solver (SVD). Multiple MCMC chains are run (one is used for inference), and convergence is checked via the Gelman-Rubin test. Finally, the set of parameters with the highest likelihood is the seed for a local non-linear minimization in the least-squares sense using the Levemberg-Marquardt algorithm.

The second otbital solution module implements an approach based on a genetic algorithm (GA). The first step consists of the generation of a population of $7 * n$ chromosomes. The fitness of each chromosome in the population is evaluated, and the selection of 'parent' chromosome pairs is made from the population according to their fitness. The next step includes the iterative modeling using a set of operators such as mutation, cross-over, Levemberg-Marquardt, and period scan. Finally, acceptance of new offsprings in the population is realized, and further iterations of the algorithm using the new population are carried out.

The last important element of the DU437 software module is constituted by its orbital stability analysis component. At present, a simple Hill stability criterion is applied (Marchal \& Bozis 1982). For a two-planet configuration, the system will be considered Hill-stable if the following inequality is satisfied:

$$
-\frac{2 M}{G^{2} M_{\star}^{3}} L^{2} h>1+3^{4 / 3} \frac{m_{1} m_{2}}{m_{3}^{2 / 3}\left(m_{1}+m_{2}\right)^{4 / 3}},
$$

where $M$ is the total mass of the system, $m_{1}$ and $m_{2}$ are the planet masses (the subscript 1 refers to the inner planet), $m_{3}$ is the mass of the star, $G$ is the gravitational constant, $M_{\star}=\left(m_{1} m_{2}+m_{1} m_{3}+m_{2} m_{3}\right)$, $L$ is the total angular momentum of the system, and $\mathrm{h}$ is the energy. This is sufficient to double-check dynamically pathologic (while potentially correct in a $\chi^{2}$ sense!) solution sets (e.g., orbit-crossing due to $e \sim 1.0$ ) obtained by the MCMC and GA algorithms. Limited use of an N-body integrator (e.g., Burlisch-Stöer) is being tested at the time of writing.

\section{THE GAIA POTENTIAL}

Using Galaxy models, our current knowledge of exoplanet frequencies, and Gaia's best-case singlemeasurement precision (10-15 $\mu \mathrm{as})$ on bright targets $(V<13)$, Casertano et al. (2008) have shown how Gaia's main strength will be its ability to measure astrometrically actual masses and orbital parameters for possibly thousands of giant planets, and to determine the degree of coplanarity in possibly hundreds of multiple-planet systems. Its useful horizon for planet detection (encompassing $\approx 3 \times 10^{5}$ mainsequence F-G-K stars) extends as far as the nearest star-forming regions (e.g., Taurus at $d \approx 140 \mathrm{pc}$ ) for systems with massive giant planets $\left(\mathrm{M}_{\mathrm{p}}>2-3 \mathrm{M}_{\mathrm{J}}\right)$ on $1<a<4 \mathrm{AU}$ orbits around solar-type hosts, 


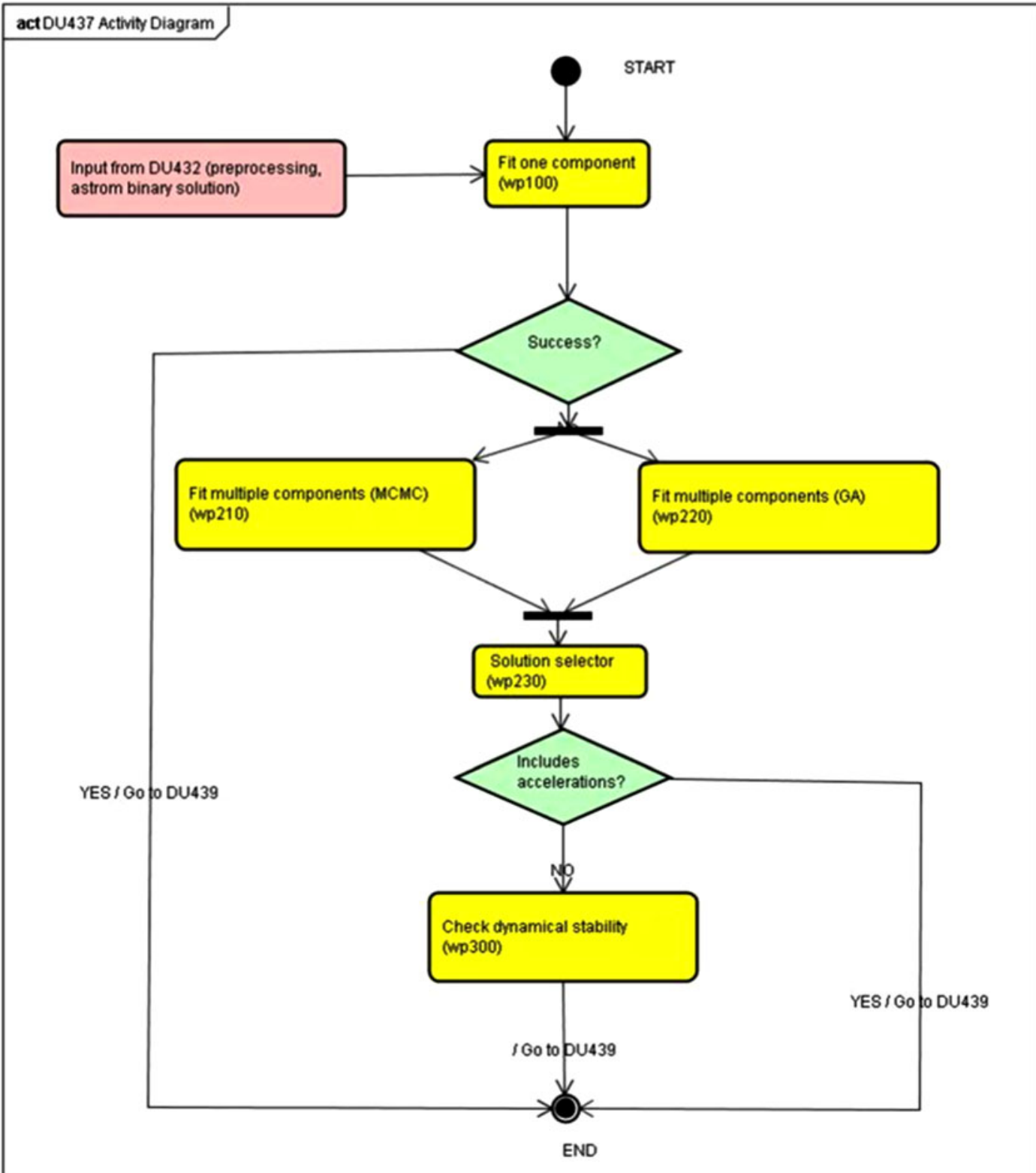

Figure 1. DU 437 Activity Diagram.

and out to $d \simeq 30$ pc for Saturn-mass planets with similar orbital semi-major axes around late-type stars (see Fig. 2).

As a result, the actual impact of Gaia measurements in exoplanets science is broad, and rather structured. The Gaia data have the potential to: a) significantly refine our understanding of the statistical properties of extrasolar planets; b) help crucially test theoretical models of gas giant planet formation and migra- tion; c) achieve key improvements in our comprehension of important aspects of the formation and dynamical evolution of multiple-planet systems; d) aid in the understanding of direct detections of giant extrasolar planets; e) provide important supplementary data for the optimization of 


\section{Hot Planets and Cool Stars}

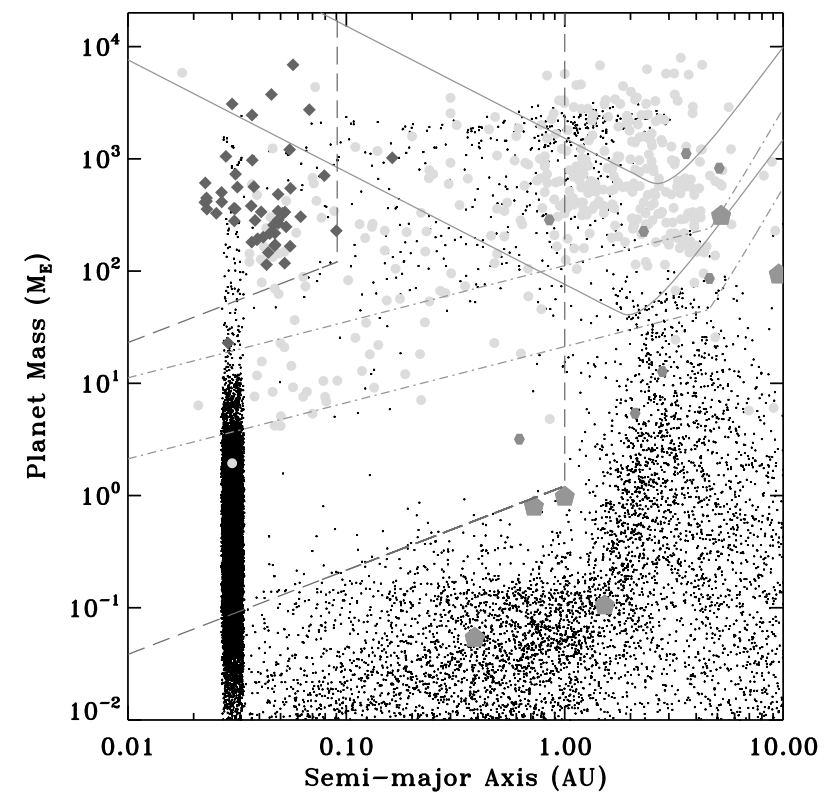

Figure 2. Exoplanets discovery space in the $M_{p}-a$ plane for the astrometric, Doppler, and transit techniques. Detectability curves are defined on the basis of a 3- $\sigma$ criterion for signal detection. The upper and lower solid curves are for Gaia astrometry with $\sigma_{\mathrm{A}}=10 \mu \mathrm{as}$, assuming a $1-M_{\odot} \mathrm{G}$ dwarf primary at $200 \mathrm{pc}$ and a $0.4-M_{\odot} \mathrm{M}$ dwarf at $25 \mathrm{pc}$. The survey duration is set to $5 \mathrm{yr}$. The radial velocity curves (dashed-dotted lines) assume $\sigma_{\mathrm{RV}}=$ $3 \mathrm{~m} \mathrm{~s}^{-1}$ (upper curve) and $\sigma_{\mathrm{RV}}=1 \mathrm{~m} \mathrm{~s}^{-1}$ (lower curve), $M_{\star}=1 M_{\odot}$, and 10-yr survey duration. For visible-light transit photometry (long-dashed curves), the assumptions are $\sigma_{V}=5 \times 10^{-3} \mathrm{mag}$ (upper curve) and $\sigma_{V}=1 \times 10^{-5} \mathrm{mag}$ (lower curve), $S / N=9, M_{\star}=1 M_{\odot}, R_{\star}=1 R_{\odot}$, uniform and dense (>>1000 datapoints) sampling. The light-grey circles indicate the inventory of Doppler-detected exoplanets as of June 2011. Transiting systems are shown as dark-grey filled diamonds, while the grey hexagons are planets detected by microlensing. Solar System planets are also shown as large grey pentagons. The small black crosses represent a theoretical distribution of masses and final orbital semi-major axes from Ida \& Lin (2008).

the target selection for future observatories aiming at the direct detection and spectral characterization of habitable terrestrial planets. For a review, see Sozzetti (2010).

The wide range of applications to exoplanets science is such that Gaia data can be seen as an ideal complement to (and in synergy with) many ongoing and future observing programs devoted to the indirect and direct detection and characterization of planetary systems, both from the ground and in space, and across a broad range of wavelengths. Gaia will contribute critically, for example, to the definition of input catalogues for proposed quasi-all-sky photometric transit surveys (PLATO, TESS); it will inform ground-based direct imaging programs (e.g., SPHERE/VLT, PCS/E-ELT) and spectroscopic characterization projects (e.g., EChO, FINESSE) about the epoch and location of maximum brightness of (primarily non transiting) exoplanets, in order to estimate their optimal visibility, and will help in the modeling and interpretation of giant planets' phase functions and light curves. Another critical aspect will concern the large effort in terms of ground-based follow-up activities to improve the characterization of astrometrically detected systems (and possibly those found transiting by Gaia photometry). For example, high-precision radial-velocity campaigns (both at visible and infrared wavelengths) will be a necessary complement, with the three-fold aim of improving the phase sampling of the astrometric orbits found by Gaia, extending the time baseline of the observations (to put stringent constraints on or actually characterize long-period companions), and search for additional, low-mass and/or short-period components which might have been missed by Gaia due to lack of sensitivity. 


\section{EPJ Web of Conferences}

\subsection{Synergies in the making: The Gaia survey of nearby $M$ dwarfs}

Cool, nearby M dwarfs within a few tens of parsecs from the Sun are becoming the focus of dedicated experiments in the realm of exoplanets astrophysics. This is due to the shift in theoretical paradigms in light of new observations, and to the improved understanding of the observational opportunities for planet detection and characterization provided by this sample. Gaia, in its all-sky survey, will deliver precision astrometry for a magnitude-limited $(V=20)$ sample of $\mathrm{M}$ dwarfs, providing an inventory of cool nearby stars with a much higher degree of completeness (particularly for late sub-types) with respect to currently available catalogs.

I present here preliminary findings of a simulation experiment aimed at gauging the Gaia potential for precision astrometry of exoplanets orbiting a sample of known, nearby dM stars (Lépine 2005). Gaia sensitivity thresholds are expressed as a function of system parameters and in view of the latest mission profile, including the most up-to-date astrometric error model. The simulations also provide insight on the capability of high-precision astrometry to reconstruct the underlying orbital elements and mass distributions of the generated companions. These results will help in evaluating the complete expected Gaia planet population around late-type stars.

The synergy between the Gaia data on nearby M dwarfs and other ground-based and space-borne programs for planet detection and characterization is also investigated, with a particular focus on: a) the potential for Gaia to precisely determine the orbital inclination, which might indicate the existence of transiting long-period planets; b) the ability of Gaia to carefully predict the ephemerides of (transiting and non-transiting) planets around $\mathrm{M}$ stars; and c) its potential to help in the precise determination of the emergent flux, for systematic spectroscopic characterization of their atmospheres with dedicated observatories in space, such as EChO.

\subsubsection{Simulation scheme}

The basis is constituted by the Casertano et al. (2008) simulation setup. This has been updated by including the lates Gaia scanning law, for a nominal $T=5 \mathrm{yr}$ mission duration. The most up-todate error model as a function of Gaia G-band mag has been utilized, with the exclusion of the presently envisioned gate scheme (affecting only some $20 \%$ of bright $(G<12) \mathrm{M}$ dwarfs). Singlemeasurement errors are typically $\sigma_{m} \sim 100 \mu$ as. The actual list of targets encompasses $3150 \mathrm{M}$ dwarfs $\left(0.09-0.6 M_{\odot}\right)$ within 33 pc from the Sun from the LSPM-North Catalog (Lpine 2005), with average $G \sim 14.0$ mag. One planet was generated around each star, with mass $M_{p}=1 M_{J}$, orbital period $P<3 T$, and moderate eccentricities $(e<0.6)$. All other orbital elements were uniformly distributed within their respective ranges.

\subsubsection{Preliminary results}

The main findings in this experiment can be summarized as follows: 1) For detected giant planets with periods in the range $0.2-5 \mathrm{yr}$ (i.e., with accurately determined masses and orbits), inclination angles will be determined with enough precision $(<1 \%$, see Fig. 3, left panel) so that it will be possible to identify long-period planets which are likely to transit; 2$)$ for well-sampled orbits $(P<T)$, the uncertainties on planetary ephemerides, separation $\varrho$ and position angle $\vartheta$, will degrade at typical rates of $\Delta \varrho<0.01 \mathrm{AU} / \mathrm{yr}$ and $\Delta \vartheta<1 \mathrm{deg} / \mathrm{yr}$, respectively. These are over an order of magnitude smaller than the degradation levels attained by present-day ephemerides predictions based on milli-arcsec precision HST/FGS astrometry (Benedict et al. 2006); 3) Planetary phases will be measured with typical uncertainties $\Delta \alpha$ of a few degrees, resulting (assuming a simple purely scattering atmosphere) in average errors on the phase function $\Delta \Phi(\alpha) \approx 0.1$, and expected uncertainties in the determination of the emergent flux of wide-separation ( $a>0.3 \mathrm{AU}$ ) giant planets of $\sim 15 \%$ (Fig. 3, right panel). 


\section{Hot Planets and Cool Stars}
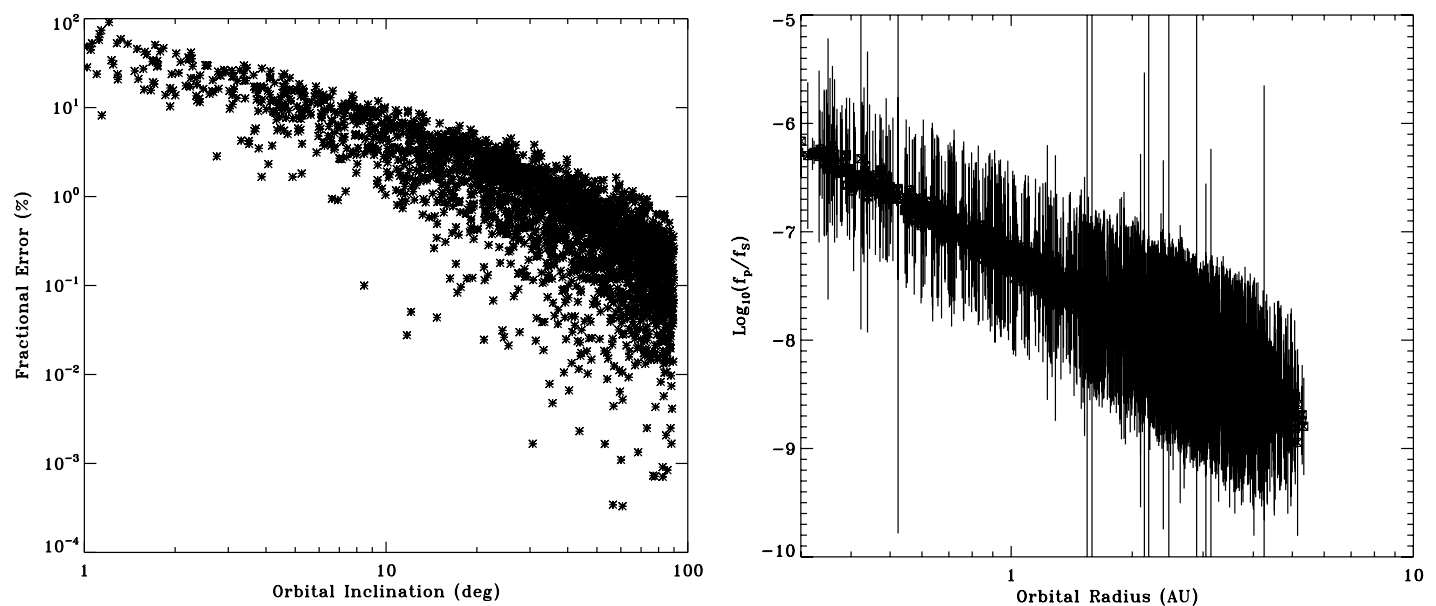

Figure 3. Left: fractional error on the inclination angle $i$ as a function of $i$ itself. Right: predicted planetary emergent flux (and uncertainty) as a function of orbital separation from the $\mathrm{M}$ dwarf primary.

Finally, it is worth pointing out that for the entire stellar sample used in this analysis Gaia will deliver exquisitely precise direct distance estimates (for a typical $V=14$ mag target at $d \sim 20 \mathrm{pc}$, one infers a parallax determination with a precision of $\approx 0.1 \%$ !). Such measurements will allow to accurately re-calibrate the absolute luminosities of stars at the bottom of the main sequence, thus enabling, by comparison with improved models of stellar evolution, the accurate $(<3-5 \%)$ determination of accurate stellar radii for the whole sample.

These results help to quantify the actual relevance of the Gaia observations of the large sample of nearby $\mathrm{M}$ dwarfs for in a synergetic effort to optimize the planning and interpretation of follow-up/characterization measurements of the discovered systems by means of transit survey programs (e.g., MEarth, APACHE, NGTS), and upcoming and planned ground-based as well as space-borne observatories for direct imaging (e.g., SPHERE, PCS) and simultaneous multi-wavelength spectroscopy (e.g., EChO, FINESSE).

\section{SUMMARY AND CONCLUDING REMARKS}

The largest compilation of high-accuracy astrometric orbits of giant planets, unbiased across all spectral types up to $d<200 \mathrm{pc}$, will allow Gaia to crucially contribute to several aspects of planetary systems astrophysics (formation theories, dynamical evolution), in combination with present-day and future extrasolar planet search programs.

In this paper, I have briefly summarized the status of the Gaia mission now that we are less than 1 year from launch. Next, I have discussed some of the relevant issue related to the robust assessment of astrometric orbits of planetary systems detected by Gaia, and described the DU437 software module in charge of providing (single and multiple) orbital solutions for exoplanets within the context of the Gaia data processing pipeline. Then, I have presented preliminary results on an investigation of the synergy between Gaia astrometry on an actual sample of nearby $\mathbf{M}$ dwarfs and other ground-based and space-borne programs for exoplanet characterization, focusing on: a) the potential for Gaia to precisely determine the orbital inclination, which might indicate the existence of transiting long-period planets; b) the ability of Gaia to carefully predict the ephemerides of (transiting and non-transiting) planets around $\mathrm{M}$ stars; and c) its potential to help in the precise determination of the emergent flux, for systematic spectroscopic characterization of their atmospheres with dedicated observatories in space. 
EPJ Web of Conferences

The author wishes to thank M.G. Lattanzi, R. Morbidelli, G. Micela, G. Tinetti, P. Giacobbe and the colleagues of (DPAC CU4) DU437 D. Ségransan, D. Sosnowska, and N. Rambaux for their help, discussions, and inputs.

\section{References}

[1] Benedict G.F., et al., AJ, 132, 2206 (2006)

[2] Casertano S., Lattanzi M.G., Sozzetti A., et al., A\&A, 482, 699 (2008)

[3] Forveille T., et al., A\&A submitted (arXiv:1109.2505) (2011)

[4] Green R.M., Spherical Astronomy (Cambridge University Press, New York, 1985)

[5] Hatzes A.P., et al., ApJ, 743, 75 (2011)

[6] Lépine S., AJ, 130, 1680 (2005)

[7] Marchal C., \& Bozis G., Celest. Mech., 26, 311 (1982)

[8] Perryman M.A.C., et al., A\&A, 369, 339 (2001)

[9] Sozzetti A., PASP, 117, 1021 (2005)

[10] Sozzetti A., EAS Pub. Ser., 42, 55 (2010)

[11] Sozzetti A., EAS Pub. Ser., 45, 273 (2011) 